\title{
A modified finite volume method incorporating radial basis functions for simulating diffusion
}

\author{
T. J. Moroney* $\quad$ I. W. Turner ${ }^{\dagger}$
}

(Received 2 December 2004, revised 15 April 2005)

\begin{abstract}
The finite volume method is the favoured numerical technique for solving (possibly coupled, nonlinear, anisotropic) diffusion equations. The method transforms differential equations into a system of nonlinear, algebraic equations through the process of discretisation. The accuracy of this discretisation determines to a large extent the accuracy of the final solution. We present a new method of discretisation which is designed to achieve high accuracy without imposing excessive computational requirements. The method employs radial basis functions as a means of local gradient interpolation. When combined with high order Gaussian quadrature integration methods, the interpolation based on radial basis functions produces an efficient and accurate
\end{abstract}

*School of Mathematical Sciences, Queensland University of Technology, Brisbane, Australia. mailto:t.moroney@qut.edu.au

${ }^{\dagger}$ School of Mathematical Sciences, Queensland University of Technology, Brisbane, Australia. mailto:i.turner@qut.edu.au

See http://anziamj.austms.org.au/V46/CTAC2004/Moro for this article, (C) Austral. Mathematical Soc. 2005. Published June 9, 2005. ISSN 1446-8735 
discretisation. The resulting nonlinear, algebraic system is solved efficiently using a Jacobian-free Newton-Krylov method. Information obtained from the Newton-Krylov iterations is used to construct an effective preconditioner in order to reduce the number of nonlinear iterations required to achieve an accurate solution. Results to date have been promising, with the method giving accuracy several orders of magnitude better than simpler methods based on shape functions.

\section{Contents}

1 Introduction

C460

2 Finite volume method

C460

3 Gaussian quadrature

C462

4 Interpolation

C462

4.1 Shape functions . . . . . . . . . . . . . . . C463

4.2 Radial basis functions . . . . . . . . . . . . . . . C C464

4.2.1 Gradient evaluation . . . . . . . . . . . . . . C464

4.2.2 Improving accuracy near boundaries . . . . . . . C C465

5 Solution of nonlinear system

C466

5.1 Preconditioning . . . . . . . . . . . C C467

6 Numerical experiments

C467

6.1 Test problem ...................... C467

6.2 Results and discussion . . . . . . . . . . . . . . C468

7 Conclusions

C470

References

C470 


\section{Introduction}

The finite volume method is a numerical method for solving partial differential equations. It is particularly well suited for solving problems involving diffusion. When using the finite volume method in this way, an integral involving the diffusive flux component must be evaluated numerically. This requires both a means of numerical integration, and a means of gradient evaluation at the integration points. The accuracy of these two components has a large bearing on the overall accuracy of the finite volume method.

We investigate the effectiveness of using radial basis functions as a means of interpolation, coupled with Gaussian quadrature as a means of numerical integration. This approach can result in highly accurate finite volume discretisations, meaning that accurate solutions can be obtained using a much coarser mesh than is possible when using simpler discretisation methods.

By using the method of radial basis functions as an extension of shape function-based methods, an accurate solution can be obtained in fewer iterations than when using the method on its own. Furthermore, the method of shape functions provides useful information that can be used to precondition the underlying linear system based on radial basis functions.

Some numerical experiments are presented that demonstrate the high accuracy of the new method, compared to that of shape functions.

\section{Finite volume method}

The finite volume method is based on a discretisation of the original partial differential equation. To this end, it employs a mesh - a geometric structure consisting of a set of nodes, and the corresponding connections between the nodes to form elements. In this work, the mesh generation software 


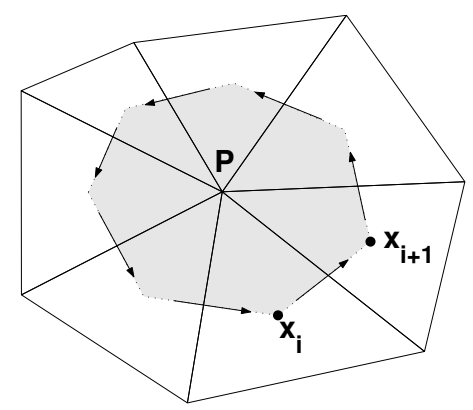

(a)

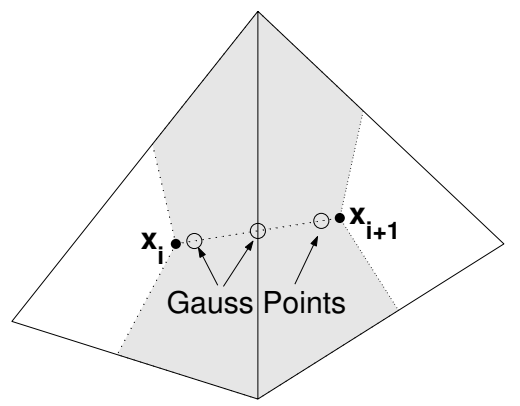

(b)

Figure 1: (a) Forming control volumes by joining element centroids. (b) Three-point Gaussian quadrature over a control volume face

EasyMesh $^{1}$ generates the unstructured, triangular mesh.

The finite volume method discretises the original conservation law by forming so-called control volumes around each node in the mesh and integrating over each control volume to produce a set of discrete function values at the nodes. Figure 1(a) shows how the control volumes are formed by joining element centroids.

To illustrate the finite volume discretisation strategy, consider the following steady-state diffusion equation

$$
\boldsymbol{\nabla} \cdot(\mathbf{D} \boldsymbol{\nabla} \varphi)+S=0 .
$$

This partial differential equation is transformed into two-dimensional controlvolume form by integrating over each control volume $V_{P}$ and applying the divergence theorem

$$
\sum_{i=0}^{n-1} \int_{\mathbf{x}_{i}}^{\mathbf{x}_{i+1}} \mathbf{D} \nabla \varphi \cdot \hat{\mathbf{n}} d s+\Delta A_{P} \bar{S}=0,
$$

${ }^{1}$ See http://www-dinma.univ.trieste.it/nirftc/research/easymesh 
where $\bar{S}=\frac{1}{\Delta A_{P}} \int_{V_{P}} S d V$ is the volume average of $S$. Equation (2) is exact since no approximation has been made to this point of the derivation. Solving this equation requires approximating the line integral $\int_{\mathbf{x}_{i}}^{\mathbf{x}_{i+1}} \mathbf{D} \boldsymbol{\nabla} \varphi \cdot \hat{\mathbf{n}} d s$ using an appropriate quadrature rule. The accuracy of this approximation has a large bearing on the overall accuracy of the finite volume method. We investigate approximating these integrals using Gaussian quadrature.

\section{Gaussian quadrature}

Numerical quadrature methods approximate the integral in (2) by a sum of weights times integrand values

$$
\int_{\mathbf{x}_{i}}^{\mathbf{x}_{i+1}} \mathbf{D} \nabla \varphi \cdot \hat{\mathbf{n}} d s \approx \sum_{j} w_{j}[\mathbf{D} \nabla \varphi \cdot \hat{\mathbf{n}}]_{\mathbf{x}_{j}} .
$$

Gaussian quadrature is a higher order method than the commonly used midpoint rule. To apply it to (3), we use the parameterisation

$$
\mathbf{x}(t)=\frac{1}{2}(1-t) \mathbf{x}_{i}+\frac{1}{2}(1+t) \mathbf{x}_{i+1}, \quad-1 \leq t \leq 1 .
$$

This three-point scheme is applied over the length of the control volume face as illustrated in Figure 1(b). It can be shown [4] that whereas the midpoint rule is $\mathcal{O}\left(h^{2}\right)$, three-point Gaussian quadrature is $\mathcal{O}\left(h^{6}\right)$, where $h$ is the length of (in this case) the control volume face.

\section{Interpolation}

To compute the line integral (3) using the three-point Gaussian quadrature technique outlined in Section 3, the values of the integrand $\mathbf{D} \boldsymbol{\nabla} \varphi \cdot \hat{\mathbf{n}}$ are 
needed at points on the control volume face. Recall that as a result of the finite volume discretisation, only the values of $\varphi$ are available at the node points in the mesh. These values must be used to reconstruct the required gradient $\boldsymbol{\nabla} \varphi$ at the control volume face. We discuss two methods to achieve this: the well-known shape function method, and a novel method based on radial basis functions.

\subsection{Shape functions}

A popular method of interpolation in the finite volume literature is borrowed from finite element theory. The method of shape functions uses the values of $\varphi$ on the three vertices of a triangular element to compute a constant interpolation of $\boldsymbol{\nabla} \varphi$ within the element [3]:

$$
\boldsymbol{\nabla} \varphi=\sum_{i=1}^{3} N_{i} \varphi_{i} .
$$

Finite volume methods that employ these shape functions are often called control volume-finite element, or CV-FE, methods. For problems where the gradient does not vary greatly over small regions this approach can be satisfactory. However, in many problems, there are regions where the gradient varies significantly and using shape functions will not adequately capture this behaviour.

Some work has been done (see for example [6]) on extending CV-FE methods of flux approximation to second and higher orders of accuracy. Typically, a least-squares approach is used to formulate a higher-degree interpolating polynomial. Rather than extend or improve upon this work, it was decided to investigate alternative methods of interpolation that might also yield highorder gradient approximations. 


\subsection{Radial basis functions}

The method of radial basis functions, or RBFs, is a scattered data interpolation method in $\mathbb{R}^{n}[9]$. Given a set of nodes $\left\{\mathbf{x}_{1}, \mathbf{x}_{2}, \ldots, \mathbf{x}_{N}\right\}$ and corresponding function values $\left\{\varphi_{1}, \varphi_{2}, \ldots, \varphi_{N}\right\}$, the RBF interpolating function $s$ is

$$
s(\mathbf{x})=\sum_{j=1}^{N} \lambda_{j} \phi\left(\left\|\mathbf{x}-\mathbf{x}_{j}\right\|\right)+\sum_{k=0}^{\operatorname{dim}\left(\pi_{m}^{n}\right)} c_{k} p_{k}(\mathbf{x}),
$$

where the $\lambda_{j}$ and $c_{k}$ are determined by the conditions

$$
s\left(\mathbf{x}_{j}\right)=\varphi\left(\mathbf{x}_{j}\right), \quad j=1,2, \ldots, N,
$$

and

$$
\sum_{j=1}^{N} \lambda_{j} p_{k}\left(\mathbf{x}_{j}\right)=0, \quad k=0,1, \ldots, \operatorname{dim}\left(\pi_{m}^{n}\right),
$$

where $\pi_{n}^{m}$ denotes the space of all polynomials in $n$ variables up to degree $m$ and the $p_{k}$ are the standard basis polynomials for this space. The choices for $\phi(r)$ used in this study are the thin plate spline, $\phi(r)=r^{2} \log (r)$ and the multiquadric, $\phi(r)=\sqrt{c^{2}+r^{2}}$. The multiquadric function includes a free parameter $c$. The value chosen for this parameter affects the accuracy of the resultant RBF interpolation. Numerical experiments determined a value that resulted in accurate interpolations.

\subsubsection{Gradient evaluation}

Powell [9] showed that the function $s$ of (6) is differentiable for both the thin plate spline and the multiquadric on any region of $\mathbb{R}^{n}$ that excludes the interpolation points. Thus from (6),

$$
\boldsymbol{\nabla} s=\sum_{j=1}^{N} \lambda_{j}\left(\frac{\mathbf{x}-\mathbf{x}_{j}}{\left\|\mathbf{x}-\mathbf{x}_{j}\right\|}\right) \phi^{\prime}\left(\left\|\mathbf{x}-\mathbf{x}_{j}\right\|\right)+\sum_{k=0}^{\operatorname{dim}\left(\pi_{m}^{n}\right)} c_{k} \boldsymbol{\nabla} p_{k}(\mathbf{x}) .
$$




\subsubsection{Improving accuracy near boundaries}

It is well known that the accuracy of RBF-based interpolations is poorest near the convex hull of the set of nodes [5]. We overcome this problem in two ways:

1. Using one interpolation per element, as with shape functions;

2. Incorporating boundary information into the interpolation.

The method of shape functions fits a function $s$ that is valid only over a given triangular element. We take the same approach when using radial basis functions, and fit one function per element. Each function $s$ incorporates only a small proportion $p$ of the total number of nodes $N$. In this way, points of evaluation do not lie on or near the convex hull of the set, with the exception of points on or near the domain boundary itself. This approach also ensures the nonlinear method of Section 5 operates on a sparse Jacobian matrix, since only a local set of neighbours contribute to the computed value at a given node.

To further improve the accuracy of the interpolation, we modify the standard RBF formulation (6) so that at boundary nodes additional information taken from the corresponding boundary condition may be included. For example, the following boundary condition represents Newtonian heat conduction with an external temperature $\varphi_{\infty}$ :

$$
-\mathbf{D} \boldsymbol{\nabla} \varphi \cdot \hat{\mathbf{n}}=h\left(\varphi-\varphi_{\infty}\right) .
$$

At boundary nodes, this equation provides information about the behaviour of $\boldsymbol{\nabla} \varphi$. To make use of this information in the interpolation, we include an extra term in (6) using a second radial basis function $\phi_{2}$ (and we will now refer to the first as $\phi_{1}$ ). Assuming the first $M$ nodes are boundary nodes 
with a boundary condition such as (10), the new form for $s$ is

$$
s(\mathbf{x})=\sum_{j=1}^{N} \lambda_{j} \phi_{1}\left(\left\|\mathbf{x}-\mathbf{x}_{j}\right\|\right)+\sum_{j=1}^{M} \gamma_{j} \phi_{2}\left(\left\|\mathbf{x}-\mathbf{x}_{j}\right\|\right)+\sum_{k=0}^{\operatorname{dim}\left(\pi_{m}^{n}\right)} c_{k} p_{k}(\mathbf{x}),
$$

where in addition to (7) and (8) we impose

$$
\boldsymbol{\nabla} s\left(\mathbf{x}_{j}\right) \cdot \hat{\mathbf{n}}=\boldsymbol{\nabla} \varphi\left(\mathbf{x}_{j}\right) \cdot \hat{\mathbf{n}}, \quad j=1,2, \ldots, M .
$$

\section{Solution of nonlinear system}

In the general nonlinear case, (2) is the Pth co-ordinate function of a nonlinear system,

$$
\mathbf{F}(\varphi)=\mathbf{0},
$$

that must be solved to obtain the values $\varphi=\left(\varphi_{1}, \varphi_{2}, \ldots, \varphi_{N}\right)^{T}$. A Jacobianfree Newton-Krylov method utilising GMRES-DR [8] is used to solve this system. The solution is obtained in three stages, using three different interpolation strategies in the evaluation of $\mathbf{F}$ :

\section{Shape functions;}

2. Radial basis functions without additional boundary information;

3. Radial basis functions with additional boundary information.

The reasons for this three-stage process are to first make use of the much cheaper shape functions to compute a solution of reasonable accuracy, while at the same time generating information used in preconditioning subsequent stages. This solution is then "corrected" using radial basis functions to obtain the final solution. The two stages of radial basis functions are necessary 
to ensure the solution is highly accurate before boundary information is introduced. Failure to ensure this may lead to divergence since the boundary information (which describes the behaviour of the exact solution) will not be applicable when the solution is far from accurate.

\subsection{Preconditioning}

We use two preconditioners in combination. The first is a matrix of specified sparsity minimising $\|\mathbf{I}-\mathbf{J M}\|_{F}$, as discussed by Carpentieri, Duff and Giraud [2]. The second is the deflation matrix of Burrage and Erhel [1] that takes eigenvalue information from GMRES-DR and forms a matrix that explicitly deflates the smallest $k$ eigenvalues. The majority of the work in forming these two preconditioners is done in stage one of the nonlinear process, under the assumption that the Jacobian matrices in all three stages will share similar spectral properties.

\section{$6 \quad$ Numerical experiments}

\subsection{Test problem}

The test problem is the steady-state heat diffusion equation

$$
\boldsymbol{\nabla} \cdot(\mathbf{D} \boldsymbol{\nabla} \mathbf{u})=-g_{0}
$$

with constant diffusion tensor $\mathbf{D}=\operatorname{diag}\left(D_{x x}, D_{y y}\right)$ and constant source $g_{0}$ on the domain $0 \leq x \leq a, 0 \leq y \leq b$. The boundaries $x=0$ and $y=0$ are insulated, and the boundaries $x=a$ and $y=b$ are subject to Newtonian cooling with external temperature $\varphi_{\infty}$. Özişik [7] gave the analytic solution of this problem. Table 1 lists the parameter values used in the experiments. 
TABLE 1: Parameter values for numerical experiments

\begin{tabular}{|c|l|c|}
\hline Parameter & Description & Value \\
\hline$D_{x x}$ & Thermal diffusivity in $x$ direction & $5.0 \mathrm{~m}^{2} \mathrm{~s}^{-1}$ \\
$D_{y y}$ & Thermal diffusivity in $y$ direction & $5.0 \times 10^{i} \mathrm{~m}^{2} \mathrm{~s}^{-1}$ \\
$g_{0}$ & Source & $10.0 \mathrm{Km}^{-2} \mathrm{~s}^{-1}$ \\
$h$ & Heat transfer coefficient & $2.0 \mathrm{Wm}^{-2} \mathrm{~K}^{-1}$ \\
$\varphi_{\infty}$ & External temperature & $20.0 \mathrm{~K}$ \\
$N$ & Number of nodes in mesh & 139 \\
$c$ & Multiquadric parameter & 3.0 \\
$p$ & Proportion of nodes used for interpolation & 0.2 \\
$s$ & Degree of polynomial term & 3 \\
\hline
\end{tabular}

Although this problem is linear, for testing purposes the full nonlinear framework outlined above was used to compute the solution. The ratio $D_{y y} / D_{x x}$ was varied from 1 to 1000 to provide an increasingly challenging numerical problem.

\subsection{Results and discussion}

Figure 2(a) shows the accuracy of the numerical solution after each of the three stages of the Newton-Krylov method. The relative error $\left\|\varphi^{e}-\varphi^{a}\right\| /\left\|\varphi^{e}\right\|$ is used, where $\varphi_{i}^{e}$ is the exact solution at node $i$ and $\varphi_{i}^{a}$ is the approximate solution at node $i$. Several features are apparent. First, the accuracy of the approximate solution diminishes as the ratio $D_{y y} / D_{x x}$ is increased. This is in agreement with the observation that the problem is most difficult numerically when this ratio is large. Second, the accuracy of the RBF-based methods is much better than that offered by the method of shape functions. Third, the inclusion of boundary information in stage three is beneficial, giving typically an order of magnitude increase in the accuracy of the solution.

The increased accuracy offered by the RBF-based methods is not with- 


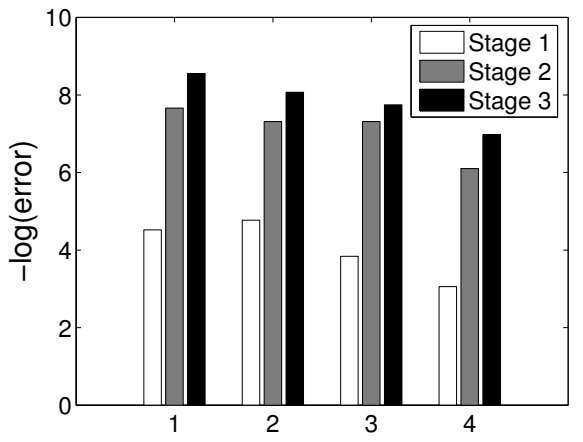

(a)

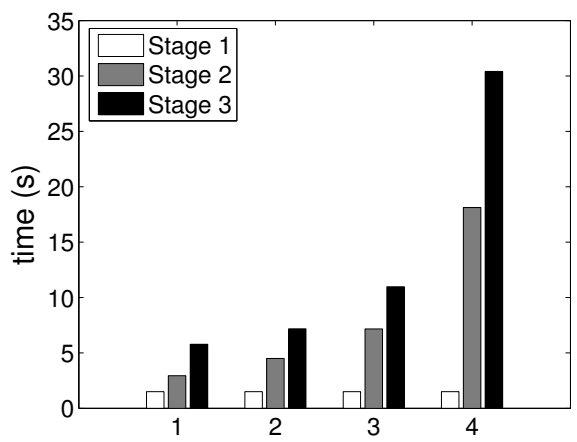

(b)

Figure 2: (a) Error after each stage. (b) Cumulative time for each stage: $1, D_{y y} / D_{x x}=1 ; 2, D_{y y} / D_{x x}=10 ; 3, D_{y y} / D_{x x}=100 ; 4, D_{y y} / D_{x x}=1000$.

TABLE 2: Number of elements needed and time taken for comparable accuracy between shape functions and RBFs

\begin{tabular}{|r|rr|}
\hline & Elements & Time(s) \\
\hline Shape functions & 5790 & 228 \\
Radial basis functions & 236 & 18 \\
\hline
\end{tabular}


out cost. Each iteration involving radial basis functions takes significantly more time than those that use shape functions. Figure 2(b) compares the cumulative time taken to reach a solution after each stage. Employing RBFs results in a much longer execution time than when using shape functions alone. However, a much more accurate solution is generated. Table 2 shows the number of elements needed in the mesh for shape functions to generate a solution of comparable accuracy to radial basis functions in the case where $D_{y y} / D_{x x}=1000$. It also shows the time taken to generate this solution. Using radial basis functions in this case allows accurate solutions to be generated in much less time than by using shape functions alone.

\section{Conclusions}

The use of radial basis functions along with three-point Gaussian quadrature in the discretisation of the finite volume method can yield accurate solutions on a much coarser mesh than is possible when using shape functions. This approach is well-suited for steady-state diffusion problems, and can be incorporated into a Newton-Krylov method for solving nonlinear partial differential equations. The use of the method as a corrector to the method of shape functions is advantageous in that it allows for an accurate solution with fewer nonlinear iterations, and for efficient preconditioning by re-using information from the shape function-based iterations. Future research will see this strategy tested on nonlinear and heterogeneous diffusion problems in a three dimensional framework.

\section{References}

[1] K. Burrage and J. Erhel. On the performance of various adaptive preconditioned GMRES strategies. Numerical Linear Algebra with 
Applications, 5(2):101-121, 1998.

http://citeseer.ist.psu.edu/362843.html C467

[2] B. Carpentieri, I. S. Duff, and L. Giraud. Sparse pattern selection strategies for robust frobenius-norm minimization preconditioners in electromagnetism. Numerical linear algebra with applications, 7:(7-8), 2000. http://citeseer.csail.mit.edu/carpentieri00sparse.html $\mathrm{C} 467$

[3] R. D. Cook. Concepts and applications of finite element analysis. Wiley, 2001. C463

[4] J. F. Epperson. An Introduction to Numerical Methods and Analysis. Wiley, 2002. C462

[5] B. Fornberg, T. A. Driscoll, G. Wright, and R. Charles. Observations on the behavior of radial basis function approximations near boundaries. Computers \& Mathematics with Applications, 43(3-5):473-490, 2002. URL C465

[6] P. Jayantha and I. W. Turner. A new higher order control-volume finite-element least-squares strategy for simulating transport in highly anisotropic media. Submitted to Journal of Computational Physics, 2002. C463

[7] M. N. Özişik. Heat Conduction. Wiley, 1980. C467

[8] R. Morgan. GMres with deflated restarting. SIAM J. Sci. Comput., 24(1):20-37, 2002.

http://epubs.siam.org/sam-bin/dbq/article/36465 C466

[9] M. Powell. The theory of radial basis function approximations in 1990, in Advances in Numerical Analysis, Vol. II: Wavelets, Subdicision Algorithms and Radial Basis Functions, pages 105-210. Oxford University Press, 1990. C464 